\title{
Dilated cardiomyopathy and ovarian dysgenesis in a patient with Malouf syndrome: A case report
}

\author{
KSENIJA GERSAK ${ }^{1}$, MARUSA STRGULC ${ }^{1}$, VOJKA GORJUP ${ }^{2}$, ZVEZDANA DOLENC-STRAZAR ${ }^{3}$, \\ VESNA JURCIC ${ }^{3}$, DANIEL J. PENNY ${ }^{4}$ and YUXIN FAN ${ }^{4}$ \\ ${ }^{1}$ Department of Obstetrics and Gynecology; ${ }^{2}$ Division of Intensive Internal Medicine, \\ Department of Internal Medicine, University Medical Center Ljubljana; ${ }^{3}$ Institute of Pathology, \\ Medical Faculty, University of Ljubljana, Ljubljana SI-1000, Slovenia; ${ }^{4}$ John Welsh Cardiovascular Diagnostic Laboratory, \\ Section of Cardiology, Department of Pediatrics, Baylor College of Medicine, Houston, TX 77030, USA
}

Received March 2, 2013; Accepted July 15, 2013

DOI: $10.3892 / \mathrm{mmr} .2013 .1669$

\begin{abstract}
Malouf syndrome is a rare congenital disorder involving the heart, genitalia, skin and skeletal characteristics. In the present study, we report on the sporadic case of a young female with dilated cardiomyopathy, hypergonadotropic hypogonadism, a small chin, bilateral blepharoptosis, marfanoid elongated fingers and hypothyroidism. Malouf syndrome may be caused by heterozygous mutations in the lamin A/C (LMNA) gene. Genetic analyses and autopsy were performed. In spite of the patient's features, sequence analysis of the coding region of the LMNA gene including exon-intron boundaries identified only one benign polymorphism: homozygous silent variant 1698C>T (H566). There is a possibility that the sequence analysis may have not detected intronic mutations or mutations in portions of the 5'- and 3 '-untranslated regions, which would confirm the clinical diagnosis.
\end{abstract}

\section{Introduction}

Malouf syndrome (1), also known as dilated cardiomyopathy-hypergonadotropic hypogonadism (DCM-HH) syndrome, is a congenital disorder. The clinical features include congestive or dilated cardiomyopathy, ovarian dysgenesis in females or primary testicular failure in males, mental retardation, broad nasal base, blepharoptosis, skin lesions, bone abnormalities and occasionally marfanoid habitus. Not all features are present in every individual case (1-5).

Correspondence to: Professor Ksenija Gersak, Department of Obstetrics and Gynecology, University Medical Center Ljubljana, 3 Slajmerjava, SI-1000 Ljubljana, Slovenia

E-mail: ksenija.gersak@mf.uni-lj.si

Key words: Malouf syndrome, LMNA gene, hypergonadotropic hypogonadism, dilated cardiomyopathy
DCM-HH may be caused by heterozygous mutations in the lamin A/C (LMNA) gene. Chen et al (6) identified three heterozygous missense mutations A57P, R133L and L140R in the LMNA gene in four patients reported to exhibit atypical Werner syndrome (adult progeria) (7). The mutations altered relatively conserved residues in LMNA.

The heterozygous missense mutation L59R, in the LMNA gene, was identified by McPherson et al (8), who noted the phenotypic similarities between their case, with cardiomyopathy and hypergonadotropic hypogonadism, and female cases previously studied by Nguyen et al (9) and Chen et al (6), described as exhibiting atypical Werner syndrome. Although these patients had a progeroid appearance, none had severe growth failure, alopecia or rapidly progressive atherosclerosis and McPherson et al (8) suggested that the phenotype represents a distinct laminopathy involving DCM-HH.

In this study, we present the case of a young female patient with DCM-HH, including the genetic analysis of the LMNA gene. The study was approved by theNational Medical Ethics Committee of the Republic of Slovenia (No.39/02/05) and written informed consent was obtained from the patient's family.

\section{Case report}

Patient history. The Caucasian female patient was the only child born to unrelated parents. The height of the patient was $165 \mathrm{~cm}$ with a weight of $50 \mathrm{~kg}$. The patient had finished upper secondary school and had no history of mumps, diabetes, surgery or radiation to the pelvic region or autoimmune disorders. The first-degree relatives of the patient had experienced normal puberties and were of normal statures. There was no family history of menstrual disorders or dysmorphic features. The patient had primary amenorrhea without the somatic stigmata of Turner's syndrome. The secondary sexual characteristics of the patient were undeveloped, including breast, pubic and axillary hair stages (Tanner stage B1, P1 and A1). The features of the patient included a small chin, bilateral blepharoptosis and marfanoid elongated fingers.

Since the age of 28 , the patient had exhibited with dyspnea, leg edemas and paroxysmal supraventricular tachycardia 


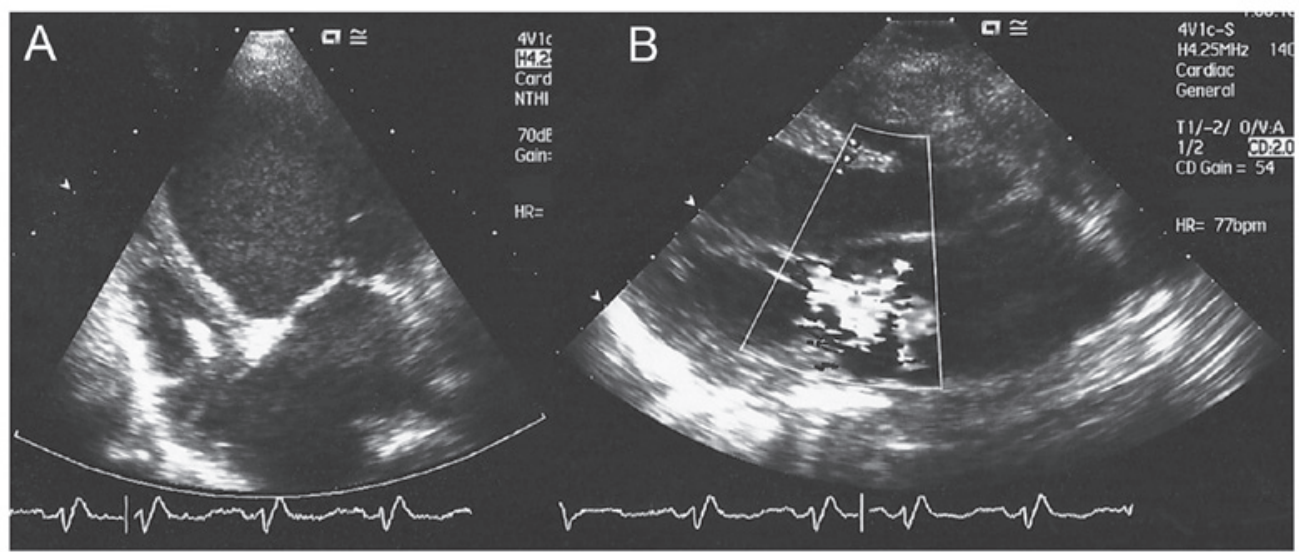

Figure 1. (A) Four-chamber apical view: enlarged left ventricle and left atrium, and normal sized right ventricle. (B) Two-dimensional parasternal view: enlarged left ventricle and moderate mitral regurgitation.

inducing syncope. Initial ultrasound evaluation indicated dilated cardiomyopathy with systolic dysfunction. X-rays revealed no evidence of bone dysplasia.

Hormonal evaluation included follicle-stimulating hormone (112.2 IU/l; menopausal range 21.7-153 IU/1), luteinizing hormone (72.5 IU/1; menopausal range 11.3-40 IU/l), estradiol $(<0.03 \mathrm{nmol} / \mathrm{l}$; menopausal range $0-0.11 \mathrm{nmol} / \mathrm{l})$ and prolactin $(9.1 \mathrm{ng} / \mathrm{ml}$; normal range $4.5-40 \mu \mathrm{g} / \mathrm{l})$. Thyroid function tests were consistent with subclinical hypothyroidism (thyroid stimulating hormone $7.7 \mathrm{mIU} / 1$, normal range $0.27-4.2 \mathrm{mIU} / \mathrm{l})$. However, a normal sized thyroid gland was detected by the ultrasound. The patient refused to receive hormone replacement therapy.

When the patient was 29 years old, a cardioverter-defibrillator was implanted due to symptomatic paroxysms of ventricular tachycardia. The patient's condition was worsening every year due to recurrent cardiac decompensation.

Admission. At the age of 33 years, the patient was admitted to the Division of Intensive Internal Medicine due to congestive heart failure. At the time of admission the patient exhibited dyspnea, orthopnea and lethargy. Major diastolic and systolic dysfunction was observed upon ultrasound examination [end-diastolic dimension (EDD) $7.7 \mathrm{~cm}$, end-systolic dimension (ESD) $6.6 \mathrm{~cm}$ and the left ventricular diastolic posterior wall dimension $(\mathrm{LVPWd}) 0.7 \mathrm{~cm}]$ with moderate mitral valve regurgitation (Figs. 1 and 2). The left ventricular ejection fraction was $10-15 \%$. On the inner wall of the left ventricle, a thrombus was observed. The patient required an intra-aortic balloon pump, in addition to inotropic and vasoactive support with noradrenalin and dobutamine. Urgent transplantation of the heart was scheduled, but the patient died on the fifth day of hospitalization due to irreversible cardiogenic shock accompanied by multiple organ failure.

Autopsy. An autopsy confirmed the diagnosis of asthenic constitution without any secondary sexual characteristics. The heart was enlarged, dilated and weighed $680 \mathrm{~g}$ (Fig. 3). The left ventricular wall and interventricular septum measured 11 and $12 \mathrm{~mm}$, respectively; and the right ventricular wall measured $3 \mathrm{~mm}$ (Fig. 4). Multiple fibrous scars were observed in the inner section of the left ventricular wall and

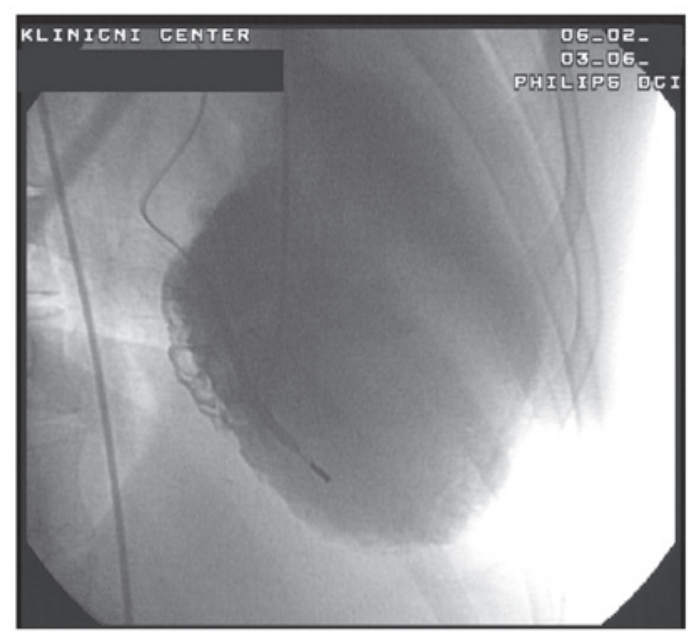

Figure 2. Cardiac ventriculography: enlarged left ventricle.

interventricular septum. Histological examination of the left ventricular myocardium revealed hypertrophied myocytes with polymorphic nuclei and multiple nucleoli (Fig. 5). Diffuse interstitial fibrosis with rare, small foci of mononuclear infiltration was observed. The endocardium was focally thickened, with parietal thrombi, the heart valves were normal and the coronary arteries were slightly atherosclerotic.

The uterus of the patient was small $(3 \times 1.5 \mathrm{~cm})$ and its cavity was covered with an atrophic endometrium and few glands. The Fallopian tubes were almost obliterated with abnormal fimbriae and there were hypoplastic streak gonads.

The thyroid gland was located on the anterior side of the neck and comprised of two symmetrical lobes and isthmus. At the microscopic level, the structure was normal.

Genetic analysis. Genomic DNA was used for polymerase chain reaction (PCR) amplification of the 12 exons contained in the coding region of the LMNA gene. The resulting PCR products were screened for mutations by bidirectional sequencing using the automated fluorescence dideoxy sequencing method. Sequence analysis identified the benign polymorphism: homozygous 1698C $>\mathrm{T}$ (H566), which is not associated with the disease. 


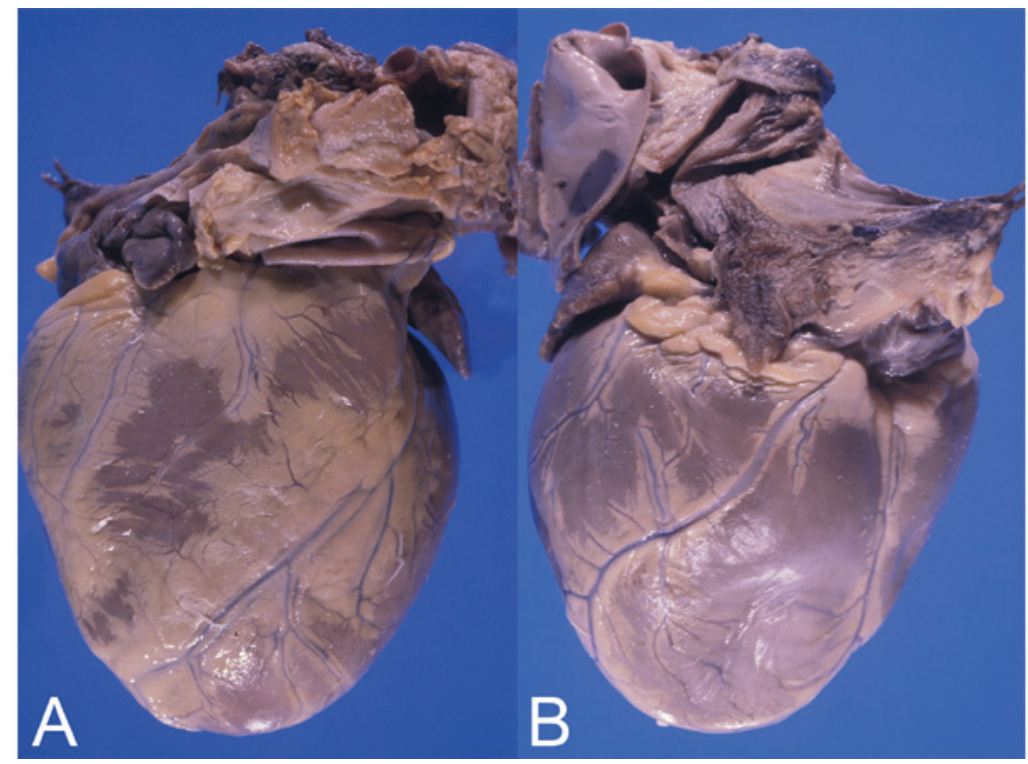

Figure 3. (A) Anterior view of the heart. (B) Posterior view of the heart.

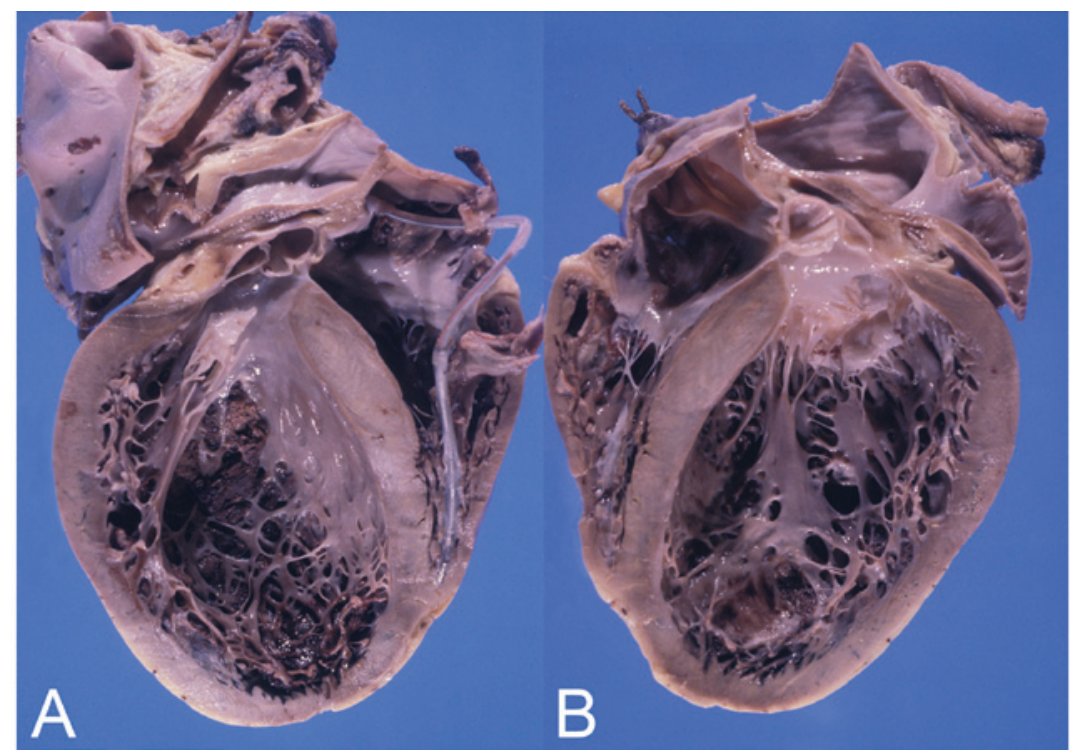

Figure 4. (A) Anterior view of the heart, longitudinal cross section, showing dilatation of both ventricles, particularly the left, with wall thinning and parietal thrombosis. The tip of the pacemaker electrode is in the apex of the right ventricle. (B) Posterior view of the heart, longitudinal cross section, showing dilatation of both ventricles, particularly the left, with wall thinning and parietal thrombosis in the left ventricle. Patchy endocardial fibrosis is observed in the right ventricle.

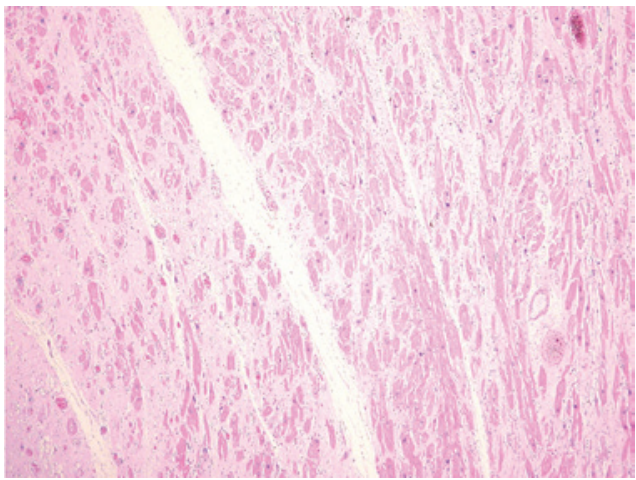

Figure 5. Histological section of the heart, with prominent interstitial fibrosis, particularly in the inner section of the wall of the left ventricle. HE staining; magnification, $\mathrm{x} 100$.

\section{Discussion}

In the present study, we report on the sporadic case of a young female with DCM-HH, facial dysmorphisms and hypothyroidism. The majority of cases with DCM-HH are born in consanguineous families. Najjar et al $(3,10)$ reported five boys in two families with primary testicular failure, mental retardation and cardiomyopathy. Brothers with cardiomyopathy and primary testicular failure were also described by Sacks et al (2) and Thomas et al (11). Malouf et al reported two sisters who presented with congestive cardiomyopathy associated with ovarian dysgenesis, bilateral ptosis and prominent nasal bones (12). The girls also had small chins and marfanoid long fingers, in a similar way to the patient in this case report. 
To the best of our knowledge, this is the second reported case of DCM-HH associated with thyroid dysfunction. Familial DCM-HH with thyroid hemiagenesis was first reported by Gursoy et al (5).

The clinical heterogeneity of the syndrome is evident. Certain patients have a more complex phenotype and it is likely that several different conditions may be identified among patients previously reported to have Malouf syndrome (8).

Molecular genetic analysis was performed for all 12 exons of the LMNA gene. The polymorphism 1698C $>$ T (H566) was detected, which had previously been registered in the National Center for Biotechnology Information Single Nucleotide Polymorphism database (http://www.ncbi.nlm.nih.gov/snp/). It is a benign polymorphism with no known association with DCM-HH. No other changes to the LMNA coding region were identified in our patient. There is the possibility that the sequence analysis may have not detected intronic mutations or mutations in portions of the 5'- and 3'-untranslated regions. Thus, this result neither confirms nor rules out a mutation in the LMNA gene.

In spite of the features of our patient, the sequence analysis of exons of the LMNA gene did not confirm the clinical diagnosis of Malouf syndrome.

\section{References}

1. Online Mendelian Inheritance in Man: Cardiomyopathy, dilated, with hypergonadotropic hypogonadism (ID no. 212112). http://www.ncbi.nlm.nih.gov/omim/212112. Accessed April 11, 2012.
2. Sacks HN, Crawley IS, Ward JA and Fine RM: Familial cardiomyopathy, hypogonadism, and collagenoma. Ann Intern Med 93: 813-817, 1980.

3. Najjar SS, Der Kaloustian VM and Ardati KO. Genital anomaly and cardiomyopathy: a new syndrome. Clin Genet 26: 371-373, 1984.

4. Narahara K, Kamada M, Takahashi Y, et al: Case of ovarian dysgenesis and dilated cardiomyopathy supports existence of Malouf syndrome. Am J Med Genet 44: 369-373, 1992.

5. Gursoy A, Sahin M, Ertugrul DT, et al: Familial dilated cardiomyopathy hypergonadotrophic hypogonadism associated with thyroid hemiagenesis. Am J Med Genet A 140: 895-896, 2006.

6. Chen L, Lee L, Kudlow BA, et al: LMNA mutations in atypical Werner's syndrome. Lancet 362: 440-445, 2003.

7. Online Mendelian Inheritance in Man: Werner syndrome; WRN (ID \#277700). http://www.ncbi.nlm.nih.gov/omim/277700. Accessed January 11, 2013.

8. McPherson E, Turner L, Zador I, et al: Ovarian failure and dilated cardiomyopathy due to a novel lamin mutation. Am J Med Genet A 149A: 567-572, 2009.

9. Nguyen D, Leistritz DF, Turner L, et al: Collagen expression in fibroblasts with a novel LMNA mutation. Biochem Biophys Res Commun 352: 603-608, 2007.

10. Najjar SS, Kaloustian VM der and Nassif SI: Genital anomaly, mental retardation, and cardiomyopathy: a new syndrome? J Pediatr 83: 286-288, 1973.

11. Thomas IT, Jewett T, Lantz P, et al: Najjar syndrome revisited. Am J Med Genet 47: 1151-1152, 1993.

12. Malouf J, Alam S, Kanj H, Mufarrij A and Der Kaloustian V: Hypergonadotropic hypogonadism with congestive cardiomyopathy: an autosomal-recessive disorder? Am J Med Genet 20: 483-489, 1985. 